\title{
Editorial: Searching for the Boundaries of Microbial Speciation in a Rapidly Evolving World
}

\author{
Eric Daniel Becraft ${ }^{1 *}$ and Andrés Moya ${ }^{2,3,4}$ \\ ${ }^{1}$ Department of Biology, University of North Alabama, Florence, AL, United States, ${ }^{2}$ Institute for Integrative Systems Biology, \\ University of Valencia and Spanish Research Council (CSIC), Valencia, Spain, ${ }^{3}$ Foundation for the Promotion of Sanitary and \\ Biomedical Research of Valencian Community (FISABIO), Valencia, Spain, ${ }^{4}$ CIBER in Epidemiology and Public Health \\ (CIBEResp), Madrid, Spain
}

Keywords: microbial diversity, microbial evolution, speciation, ecotypes, population genetics

\section{Editorial on the Research Topic}

Searching for the Boundaries of Microbial Speciation in a Rapidly Evolving World

\section{INTRODUCTION}

Whether bacteria and archaea form species-like populations has been a long-standing debate. In contrast to eukaryotic organisms, horizontal gene transfer in microorganisms can make observing vertical lineages of descent difficult. Microorganisms evolve very fast, most are difficult to culture, and the number of cells in a population and community can often be $>10^{9}$ cells $/ \mathrm{ml}$, all making studying the process of evolution and speciation difficult. There is evidence to support the idea that bacteria and archaea form cohesive sequence clusters (Jain et al., 2018; Cohan, 2019), that they form ecological species (Becraft et al., 2015; Cohan, 2017), that the rate of homologous recombination can be used as the defining species barrier (Vos and Didelot, 2009; Cadillo-Quiroz et al., 2012;

OPEN ACCESS

Edited and reviewed by:

Ludmila Chistoserdova,

University of Washington,

United States

*Correspondence: Eric Daniel Becraft

ebecraft@una.edu

Specialty section:

This article was submitted to

Evolutionary and Genomic

Microbiology,

a section of the journal

Frontiers in Microbiology

Received: 03 November 2021 Accepted: 29 November 2021 Published: 23 December 2021

Citation:

Becraft ED and Moya A (2021)

Editorial: Searching for the Boundaries of Microbial Speciation in a Rapidly Evolving World.

Front. Microbiol. 12:808595. doi: 10.3389/fmicb.2021.808595
Bobay and Ochman, 2017), and that microbial species simply do not exist due to speed of evolution and a high rate of horizontal gene transfer (Doolittle and Papke, 2006; Papke et al., 2007). Often the system or organisms analyzed effect how researchers subsequently define species, though until about 10 years ago sequencing technologies and techniques were not adequate to answer many of these questions in situ. The rapid increase in sequencing technologies, such as metagenomics and single cell genomics, is allowing researchers to once again approach this topic with higher genetic resolution.

\section{SUMMARY OF CONTRIBUTED PUBLICATIONS}

While there are varying mechanisms of genetic change and speeds of evolution, the forces acting upon genetic diversity are consistent across the tree of life. In this Research Topic, we asked researchers to share studies on a multitude of systems and taxonomic groups to observe if patterns emerge in regards to microbial species and speciation. This should lead to similarities and principles that can be applied across the tree of life and in more complex ecosystems critical to our planet, such as soils, ocean water, and the human microbiome. By understanding microbial speciation and the fundamental units within an ecosystem we can better understand how ecosystems adapt to environmental change, contribute to geochemical cycling, or cause disease.

In the article entitled "The Origin of Niches and Species in the Bacterial World", Baquero et al. examined the complexity of the niche and examine a multitude of ways that bacteria and archaea might speciate and exploit those niches as populations diversify over time. In the microbial world, the concept of niche implies that a microbial organism is present or was present in the recent past (empty niche) at a particular multidimensional environmental space. However, the microbial population interacts with the niche, creating sub-niches that are colonized by 
variant sub-populations of bacteria. Such a diversification process is compensated by the genetic interactions of sub-niche bacterial variants, leading to an evolved population able to fully exploit the expanded niche. Optimization occurs when the full carrying capacity of the niche is reached, and such perfect niche-bacteria trade-off facilitates specialization and eventually genetic isolation and speciation. In summary, the origin of species and the origin of niches are entirely intertwined.

The article entitled "Insights on the Evolutionary Genomics of the Blautia Genus: Potential New Species and Genetic Content Among Lineages," Maturana and Cárdenas used a pangenomic analysis combined with a whole-genome approach to reevaluate a previously known taxonomic group, which both discovered new species and reassigned others. Analysis of hundreds of publicly available Blautia strain genomes found 17 previously undescribed species and helped to reconstruct important gene gain/loss events during the diversification of the Blautia genus, shedding light about how this genus specialized to live in the gastrointestinal tract.

The article entitled "Comparative Genomic Insights into the Taxonomic Classification, Diversity, and Secondary Metabolic Potentials of Kitasatospora, a Genus Closely Related to Streptomyces," Li et al. used comprehensive phylogenomics and comparative genomics analyses to examine genomic differences among lineages within the genus Kitasatospora. It revealed genomic differences between the closely related genera Streptomyces and Streptacidiphilus, supporting Kitasatospora as a separate genus within the family Streptomycetaceae. Comprehensive annotation also uncovered unexpected potentials of secondary metabolism in Kitasatospora, which carries more and longer secondary metabolite biosynthetic gene clusters than the well-known antibiotic producer Streptomyces.

The article entitled "Phylogenetic Distribution of Polysaccharide-Degrading Enzymes in Marine Bacteria," Sun et al. used large-scale genomics to annotate polysaccharide-degrading enzymes in marine bacterial genomes based on a manually curated enzyme sequence database. The authors found extended phylogenetic distributions for most enzymes analyzed. Interestingly, there was substantial intra-clade diversity in the coding potential of polysaccharide-degrading enzymes, indicating diversification in the polysaccharide-utilization abilities and thus niche differentiation. The study suggested that the polysaccharide-degrading enzymes could be used as molecular markers for marine bacterial niche adaptation and speciation.

The article entitled "Host- and Species-Dependent Quasispecies Divergence of Severe Acute Respiratory Syndrome Coronavirus2 in Non-human Primate Models", Hwang et al. examined newly emerging SARS-CoV-2 variants that have been reported to cause higher morbidity and mortality than previous viruses despite enforcement of strict biosecurity and vaccination policies. Based on single nucleotide polymorphism analysis, the high intra-host, inter-host and inter-species genomic variability of SARS-CoV-2 following a short replication period were verified in Cynomolgus monkeys and Rhesus macaques infected with identical SARS-CoV2. These results are valuable not only for understanding SARS-CoV-2 variant evolution in hosts, but also for development of new diagnostics, vaccines, and therapeutics targeting SARS-CoV-2.

The article entitled "Comparative genomics of novel Agrobacterium G3 strains isolated from the International Space Station and description of Agrobacterium tomkonis sp. nov.," Singh et al. used a polyphasic approach to characterize the phenotypic and genotypic synapomorphies of a novel Agrobacterium tomkonis, showing it is a bacterial species, well-separated from previously named Agrobacterium species. A. tomkonis are diverse and have been isolated from hospital, plant rhizosphere soil, and the International Space Station surfaces. A. tomkonis specific gene functions notably relate to surface adhesion and could be involved in the ecological specificity of $A$. tomkonis, as suggested by their varied source of isolation. The ability to colonize nutrient-poor substrates, unusual for most agrobacteria, resonate with the finding that $A$. tomkonis genomes specifically carry genes involved in mediating attachment to surfaces, including production of putative adhesins and biofilm production. This suggests a particular ability of $A$. tomkonis to colonize different habitats that are even poorer in nutrients and harsher than soil, thus possibly escaping competition with other agrobacteria that are better at growing in richer environments, such as plant rhizospheres.

\section{DO SPECIES MATTER IN MICROBIAL COMMUNITIES?}

Using modern sequencing technologies and bioinformatic techniques, researchers observed patterns of microbial diversity across multiple taxa and in numerous distinct systems. Observations were made of microbial populations evolving to unique ecological niches, regardless of taxa or environment. While horizontal gene transfer can allow for niche adaptation and act as cohesive force among populations, the observed populations appeared to have diversified though selection to unique, and sometimes subtle, ecological niches. Continued research combined with improving technology will allow researchers to determine if a common species definition can ultimately be applied across all bacterial and archaeal lineages.

\section{AUTHOR CONTRIBUTIONS}

All authors listed have made a substantial, direct, and intellectual contribution to the work and approved it for publication.

\section{ACKNOWLEDGMENTS}

The authors would like to thank the many microbiologists that have contributed to the study of microbial species and speciation. EB would like to personally thank David Ward and Fred Cohan for their influence and guidance. 


\section{REFERENCES}

Becraft, E. D., Wood, J. M., Rusch, D. B., Kühl, M., Jensen, S. I., Bryant, D. A., et al. (2015). The molecular dimension of microbial species: 1. Ecological distinctions among, and homogeneity within, putative ecotypes of Synechococcus inhabiting the cyanobacterial mat of Mushroom Spring, Yellowstone National Park. Front. Microbiol. 6:590. doi: 10.3389/fmicb.2015.00590

Bobay, L.-M., and Ochman, H. (2017). Biological species are universal across life's domains. Genome Biol. Evol. 9, 491-501. doi: 10.1093/gbe/evx026

Cadillo-Quiroz, H., Didelot, X., Held, N. L., Herrera, A., Darling, A., Reno, M. L., et al. (2012). Patterns of gene flow define species of thermophilic archaea. PLoS Biol. 10:e1001265. doi: 10.1371/journal.pbio. 1001265

Cohan, F. M. (2017). Transmission in the origins of bacterial diversity, from ecotypes to phyla. Microbiol. Spectrum. 5:MTBP0014-2016. doi: 10.1128/microbiolspec.MTBP-0014-2016

Cohan, F. M. (2019). Systematics: the cohesive nature of bacterial species taxa. Curr. Biol. 29, R169-R172. doi: 10.1016/j.cub.2019.01.033

Doolittle, W. F., and Papke, R. T. (2006). Genomics and the bacterial speciation problem. Genome Biol. 7, 116.1-116.7. doi: 10.1186/gb-2006-7-9-116

Jain, C., Rodriguez-R, L. M., Phillippy, A. M., Konstantinidis, K. T., and Aluru, S. (2018). High throughput ANI analysis of $90 \mathrm{~K}$ prokaryotic genomes reveals clear species boundaries. Nat. Commun. 9:5114. doi: 10.1038/s41467-018-07641-9
Papke, R. T., Zhaxybayeva, O., Feil, E., Sommerfeld, K., Muise, D., and Doolittle, W. F. (2007). Searching for species in haloarchaea. Proc. Natl. Acad. Sci. U. S. A. 104, 14092-14097. doi: 10.1073/pnas.0706358104

Vos, M., and Didelot, X. (2009). A comparison of homologous recombination rates in bacteria and archaea. ISME J. 3, 199-208. doi: 10.1038/ismej.2008.93

Conflict of Interest: The authors declare that the research was conducted in the absence of any commercial or financial relationships that could be construed as a potential conflict of interest.

Publisher's Note: All claims expressed in this article are solely those of the authors and do not necessarily represent those of their affiliated organizations, or those of the publisher, the editors and the reviewers. Any product that may be evaluated in this article, or claim that may be made by its manufacturer, is not guaranteed or endorsed by the publisher.

Copyright (๑) 2021 Becraft and Moya. This is an open-access article distributed under the terms of the Creative Commons Attribution License (CC BY). The use, distribution or reproduction in other forums is permitted, provided the original author(s) and the copyright owner(s) are credited and that the original publication in this journal is cited, in accordance with accepted academic practice. No use, distribution or reproduction is permitted which does not comply with these terms. 